\title{
ROCK is Involved in Vimentin Phosphorylation and Rearrangement Induced by Dengue Virus
}

\author{
Shun Lei • Yan-Ping Tian • Wei-Dong Xiao • \\ Shu Li $\cdot$ Xian-Cai Rao $\cdot$ Jun-Lei Zhang $\cdot$ \\ Jie Yang $\cdot$ Xiao-Mei Hu $\cdot$ Wei Chen
}

Published online: 5 June 2013

(c) The Author(s) 2013. This article is published with open access at Springerlink.com

\begin{abstract}
Our previous study showed that dengue virus 2 (DENV2) infection induces rearrangement of vimentin into dense structures at the perinuclear area. However, the underlying mechanism of this phenomenon is poorly characterized. In the present work, we found that vimentin and Ser71 phosphorylated vimentin display similar distributions in DENV2-infected cells. DENV2 infection also induced ROCK activation and phosphorylation of vimentin at Ser71 as the DENV2 infection progressed. Furthermore, Ser71 phosphorylation and vimentin rearrangement induced by DENV2 infection were blocked by the ROCK inhibitor Y-27632. In addition, DENV2 led to endoplasmic reticulum (ER) redistribution in the perinuclear region of the host cells, which was partially blocked by pretreatment with Y-27632. Together, these data support indicate that
\end{abstract}

Shun Lei and Yan-Ping Tian have contributed equally to this work.

S. Lei $\cdot$ S. Li - X.-C. Rao - J.-L. Zhang $\cdot$ J. Yang ·

X.-M. Hu $(\bowtie) \cdot$ W. Chen $(\bowtie)$

Department of Microbiology, Third Military Medical University,

Chongqing 400038, People's Republic of China

e-mail: hxmay2008@163.com

W. Chen

e-mail: chongqingchenwei@126.com

Y.-P. Tian

Department of Histology and Embryology,

Third Military Medical University, Chongqing 400038,

People's Republic of China

W.-D. Xiao

Department of General Surgery, Xinqiao Hospital,

Third Military Medical University, Chongqing 400037,

People's Republic of China
ROCK may have a role in governing regulating vimentin and ER rearrangement during DENV2 infection. We hypothesize that DENV2 infection, via ROCK activation, induces both vimentin rearrangement and ER redistribution around the perinuclear region, which may play a structural role in anchoring DENV2 to replication sites.

Keywords Dengue virus - Vimentin - Rearrangement · Phosphorylation · ROCK $\cdot$ Endoplasmic reticulum

\section{Introduction}

Dengue virus (DENV) belongs to the Flaviviridae family and has four serotypes (DENV1-4). DENV infection causes a mild-to-debilitating febrile illness (classical dengue fever, DF) or life-threatening dengue hemorrhagic fever/ dengue shock syndrome (DHF/DSS) in tropical and subtropical regions of the world. Indeed, emerging evidence demonstrates that DENV has become a major public health problem in recent years, with approximately 50 million people infected each year and $\sim 2.5$ billion people worldwide at risk of infection [1]. Unfortunately, no DENVspecific therapies or vaccines are currently available.

It is well known that rearrangement of the cytoskeleton in host cells is closely involved in the virus life cycle, starting from virus entry to egress [2]. The roles of microfilaments and microtubules, two of the major cytoskeletal elements, have been extensively studied with respect to virus replication [3]. However, very little is known about the third cytoskeletal component, the intermediate filaments (IFs). Vimentin is the most abundant type III IF protein in various cell types [4]. A collapse of vimentin has been shown to be recruited to build a cage (vimentin cage) around viral factories in a variety of viruses, such as frog 
virus 3, African swine fever virus (ASFV), and human immunodeficiency virus (HIV) [5-7]. Our previous study revealed that after DENV2 infection, vimentin retracts from the cell periphery to the region surrounding the nucleus, suggesting that vimentin rearrangement may be involved in DENV2 infection [8]. This was confirmed by a recent study in which Kanlaya et al. [9] further show that vimentin interacts with heterogeneous nuclear ribonucleoproteins and dengue nonstructural (NS) protein 1. Although growing evidence indicates that vimentin is important for viral replication and release, the molecular mechanism underpinning the link between DENV infection and vimentin rearrangement remains unclear.

It has been reported that vimentin architecture is maintained in a dynamic state that is regulated by the balance between phosphorylation and dephosphorylation [10]. Phosphorylation of vimentin is induced by vimentin disassembly and spatial reorganization, which may contribute to the rearrangement of vimentin [11]. Sin et al. [12] further show that Rho-associated coiled coil-containing kinase (ROCK), a key serine/threonine kinase, may be responsible for the phosphorylation and subsequent rearrangement of vimentin. ROCK activation is involved in the complete collapse of the vimentin network to form a vimentin-containing arc near the perinuclear region [12]. However, little is known about the role of ROCK in vimentin arrangement induced by DENV.

Therefore, the aim of this study was to test the hypothesis that ROCK plays a critical role in regulating vimentin phosphorylation and the spatial reorientation of the vimentin network during DENV2 infection. We also investigated whether DENV2 infection-induced ROCK activation can cause a collapse of the endoplasmic reticulum (ER) into dense structures around the nucleus. Our results demonstrated that vimentin Ser71 is phosphorylated by ROCK in DENV2-infected ECV304 cells, followed by vimentin aggregation into dense structures around the perinuclear region as the DENV2 infection progresses. In addition, our data suggest that the ER but not Golgi apparatus and mitochondria is arranged during DENV2 infection in ECV304 cells. Together, the present study indicates that ROCK may play an important role in the rearrangements of vimentin, via vimentin phosphorylation, and the ER induced by DENV infection.

\section{Materials and Methods}

Cells and Virus

ECV304 cells were cultured in Dulbecco's modified Eagle's medium (DMEM, Gibco, USA) with $10 \%$ fetal bovine serum (FBS). Vero cells were cultured in minimal essential medium (MEM, Gibco) with $5 \%$ FBS. Aedes albopictus clone (C6/36) cells were cultured in RPMI 1640 (Gibco) with $10 \%$ FBS and used for propagation of DENV2.

DENV2 (strain Tr1751) was isolated from a patient with DF and kindly provided by Dr. Oya A (National Institute of Infectious Diseases, Japan). This virus was propagated in $\mathrm{C} 6 / 36$ cells and stored at $-70{ }^{\circ} \mathrm{C}$. The viral titer was determined by plaque assays using monolayers of Vero cell culture under $1 \%$ methylcellulose overlay medium.

\section{Antibodies and Reagents}

The mouse anti-vimentin monoclonal antibody was purchased from Sigma (Shanghai, China), and the p-vimentin (Ser71) antibody was purchased from MBL (Japan). The antibodies recognizing DENV2 NS1 protein and the Golgi apparatus were from Abcam (Hong Kong, China). The calnexin antibody and MitoTracker Green were obtained from Beyotime (China). The Rho-kinase inhibitor Y-27632 was purchased from Calbiochem and dissolved in sterile water. The working concentration of Y-27632 was determined using trypan blue exclusion. Cell viability was not significantly affected by drug treatment.

\section{Indirect Immunofluorescence Staining}

Double immunofluorescence staining was used to analyze the co-localization of the DENV2 NS1 glycoprotein with vimentin, the ER, and the Golgi apparatus in infected ECV304 cells. Briefly, cells were grown on glass cover slips for $24 \mathrm{~h}$ and were infected with DENV2 $(\mathrm{MOI}=1)$ or heat-inactivated DENV2 $\left(56{ }^{\circ} \mathrm{C}, 30 \mathrm{~min}\right.$, mock-infected) for $1 \mathrm{~h}$ at $37^{\circ} \mathrm{C}$. At $24 \mathrm{~h}$ after infection, the cover slips were washed and fixed with cold methanol. Nonspecific binding sites were blocked with $1 \%$ bovine serum albumin (BSA) and were incubated with primary antibodies at $4{ }^{\circ} \mathrm{C}$ overnight. After washing with PBS, the secondary antibodies were added for $1 \mathrm{~h}$ at $37{ }^{\circ} \mathrm{C}$.

To analyze the co-localization of the DENV2 NS1 glycoprotein with mitochondria in infected ECV304 cells, similar infection experiments were performed as above. At $24 \mathrm{~h}$ after infection, MitoTracker Green was added to the cells and incubated for $45 \mathrm{~min}$ at $37^{\circ} \mathrm{C}$ to allow internalization. Unbound MitoTracker Green was removed by washing the cells with PBS. Coverslips were fixed with cold methanol, and nonspecific binding sites were blocked with $1 \% \mathrm{BSA} / \mathrm{PBS}$. Then, the cells were incubated with anti-DENV2 NS1 protein antibody at $4{ }^{\circ} \mathrm{C}$ overnight, and Cy5-conjugated antibody (Beyotime, China) was added for $1 \mathrm{~h}$ at $37^{\circ} \mathrm{C}$.

To clarify the role of ROCK in vimentin reorganization during DENV2 infection, ECV304 cells were infected with DENV2 or pretreated with $10 \mu \mathrm{M} \mathrm{Y}-27632$ for $1 \mathrm{~h}$ at 

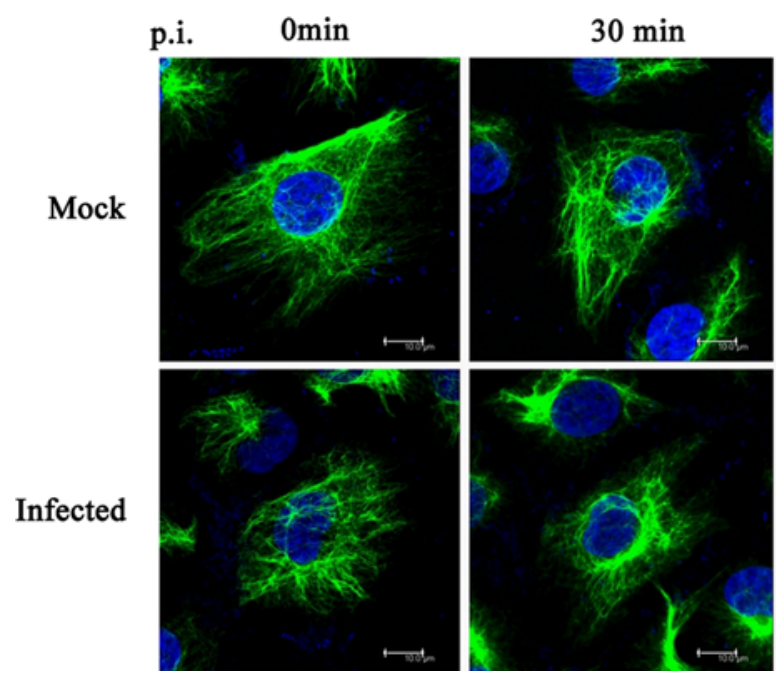

Fig. 1 Vimentin is recruited to the perinuclear region after DENV2 infection. At different time points p.i., the cells were fixed and stained with an anti-vimentin antibody. Immunostaining images show that
$1 \mathrm{~h}$

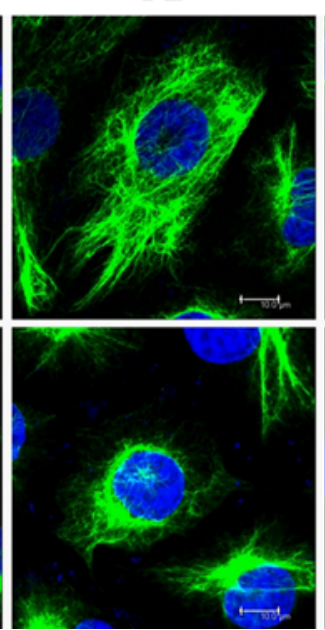

$8 \mathrm{~h}$

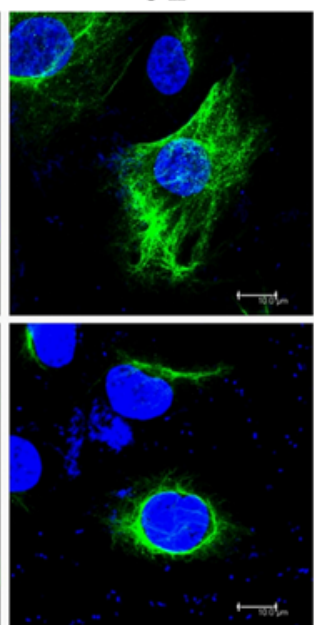

$24 \mathrm{~h}$

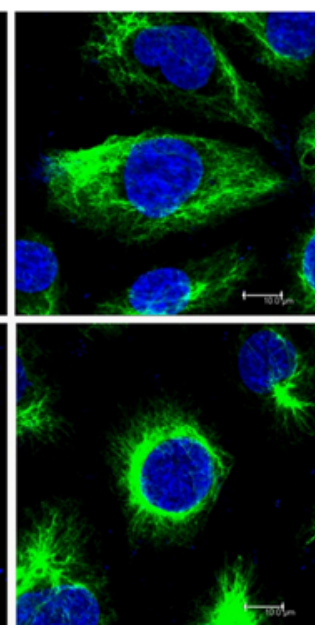

DENV2 infection induces rearrangement of vimentin as early as 30 min p.i. A marked redistribution of the organization of vimentin was observed at 1,8 , and $24 \mathrm{~h}$ p.i. Scale bars $10 \mu \mathrm{m}$

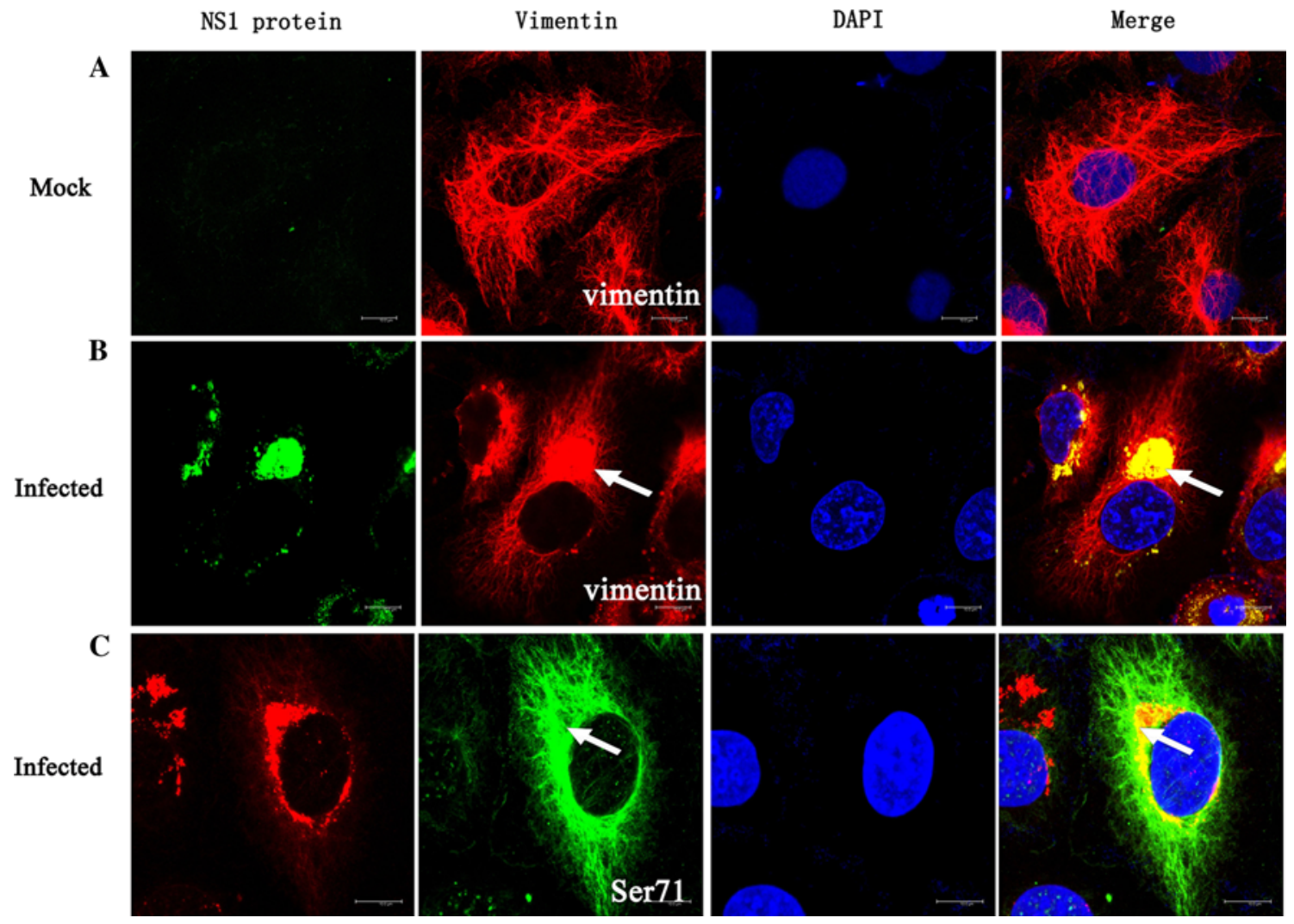

Fig. 2 DENV2 infection induced aggregation of vimentin and Ser71phosphorylated vimentin into dense structures at the perinuclear area. ECV304 cells were infected with DENV2 for $24 \mathrm{~h}$. Immunostaining shows that the characteristic distribution of vimentin in control cells

$37{ }^{\circ} \mathrm{C}$ and inoculated with active or inactivated DENV2. ECV304 cells were harvested at different time points postinfection (p.i.) in the absence or presence of Y-27632. The (a) completely changes in infected cells where vimentin (b) and phosphorylated vimentin (c) move to the perinuclear region and colocalize with DENV2 NS1 (arrows). Scale bars $10 \mu \mathrm{m}$

cells were fixed with cold methanol for 10 min at room temperature. After washing with PBS, the specimens were incubated with $1 \% \mathrm{BSA}$ and then immunostained with 
anti-vimentin antibody overnight at $4{ }^{\circ} \mathrm{C}$. After washing with PBS, the specimens were incubated with FITClabeled secondary antibody (Sigma) for $1 \mathrm{~h}$ at $37^{\circ} \mathrm{C}$.

To test effect of Y-27632 on DENV2 infection-induced ER rearrangement, similar infection experiments were performed on cells, and then $10 \mu \mathrm{M}$ Y-27632 was added to the medium after adsorption. At 24 h p.i., double-staining was performed to analyze the distribution of the ER.

All specimens were incubated with DAPI nuclear stain solution (Sigma) for $5 \mathrm{~min}$. All cell cultures were analyzed using a confocal laser scanning microscope (Leica TCS SP5, Germany).

\section{ROCK Activity Assay}

A commercially available enzyme-linked immunosorbent assay (ELISA)-based ROCK activity assay kit (Cell Biolabs, USA) was used to measure ROCK activity. ECV304 cells were incubated with DENV2 $(\mathrm{MOI}=1)$ or heat-inactivated virus $\left(56^{\circ} \mathrm{C}\right.$ for $30 \mathrm{~min}$, mock infection) for $1 \mathrm{~h}$ at $4{ }^{\circ} \mathrm{C}$ to allow attachment to occur. Then, the temperature was shifted to $37{ }^{\circ} \mathrm{C}$. ECV304 cells were harvested at different time points p.i. and tested for ROCK activation according to the manufacturer's instructions.

Western Blotting for Phosphorylation of Vimentin Ser71

ECV304 cells in 6-well plates were infected with DENV2 or pretreated with $10 \mu \mathrm{M} \mathrm{Y}-27632$ for $1 \mathrm{~h}$ and infected with DENV2. Cells were harvested and lysed in RIPA buffer at various time points after DENV2 infection in the absence or presence of Y-27632. The protein content of each sample was measured and normalized using BCA assays. Aliquots $(80 \mu \mathrm{g}$ protein) were separated on SDSPAGE gels and transferred to polyvinylidene difluoride membranes (Millipore). After blocking with $5 \%$ BSA in TBS-T (50 mM Tris- $\mathrm{HCl}, \mathrm{pH} 7.5,140 \mathrm{mM} \mathrm{NaCl}$, and $0.1 \%$ Tween), the membranes were incubated overnight at $4{ }^{\circ} \mathrm{C}$ with the following primary antibodies: mouse anti-vimentin, rat anti p-vimentin, and rabbit anti-GAPDH (Hangzhou Goodhere Biotechnology, China). The membranes were washed, incubated with HRP-conjugated goat anti-mouse, goat anti-rat, or goat anti-rabbit (respectively) secondary antibodies (ZSGB-BIO, China) and were visualized by enhanced chemiluminescence (ECL) (Thermo, USA). The fold change was determined by using Quantity One software. Each blot was performed at least six times.
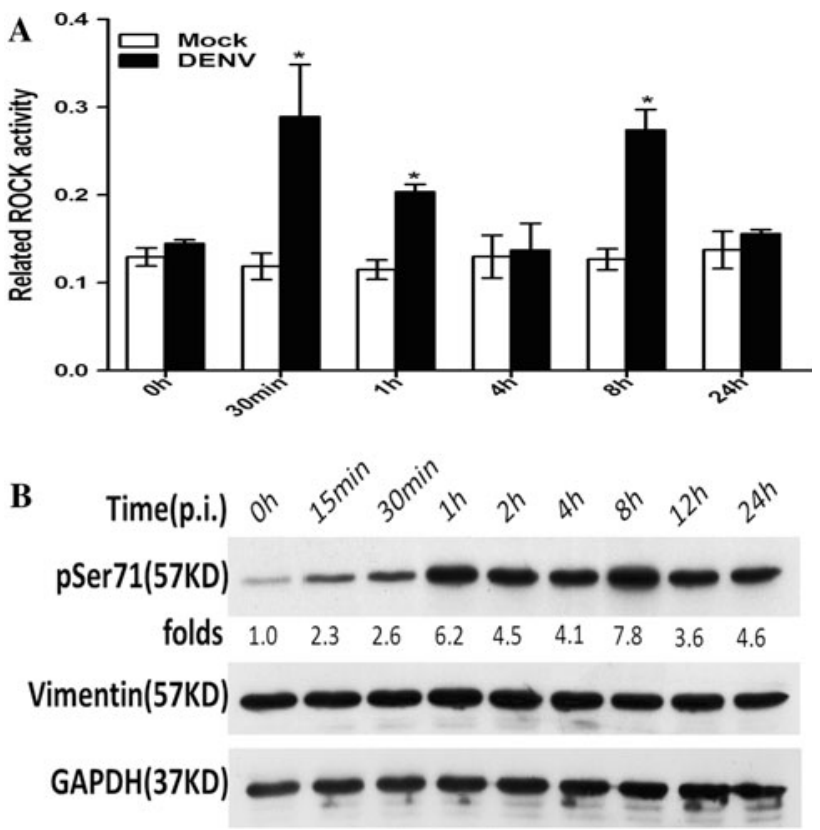

Fig. 3 DENV2 infection induced ROCK activation and phosphorylation of vimentin at Ser71. At various time points after DENV2 infection, cell lysates were analyzed by ELISA and Western blotting. a ROCK levels were measured by ELISA. A histogram depicts the change in ROCK activation. Each reaction was performed in duplicate, and each bar represents the mean + the standard deviation for three experiments. ${ }^{*} P<0.05$. b Western blot analysis was performed to detect Ser71-phosphorylated vimentin and vimentin expression, respectively. The results show that DENV2 induced an increase in the phosphorylation of vimentin at Ser71 from $15 \mathrm{~min}$ to $24 \mathrm{~h}$, with a maximum increase of 7.8-fold above control cells at $8 \mathrm{~h}$ p.i. Each blot represents data from a minimum of six separate experiments

\section{Results}

Vimentin was Recruited to the Perinuclear Region in the Early Stage of DENV2 Infection

Although recent reports demonstrate that vimentin is involved in DENV infection and replication in host cells, very few details about the precise timing of the recruitment of vimentin after DENV infection have been revealed [8, 9]. Therefore, ECV304 cells were fixed at different time points p.i. to observe the dynamic arrangement of vimentin induced by DENV2 infection. In uninfected cells, vimentin formed an extended network, from the perinuclear region to the cell periphery. In contrast, perinuclear condensation of vimentin occurred progressively within DENV2-infected cells, detectable as early as $30 \mathrm{~min}$ p.i. A substantial effect on vimentin redistribution was also observed at 8 and $24 \mathrm{~h}$ p.i. (Fig. 1). These data suggested that the rearrangement of vimentin may be an early event in the DENV2 infection process. 
A p. i. $\quad 0 \mathrm{~h}$
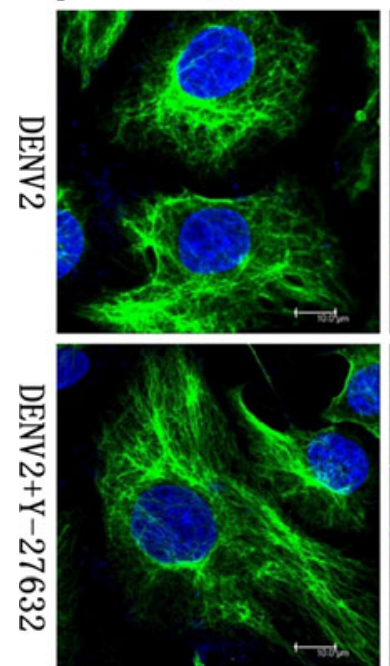

$30 \mathrm{~min}$
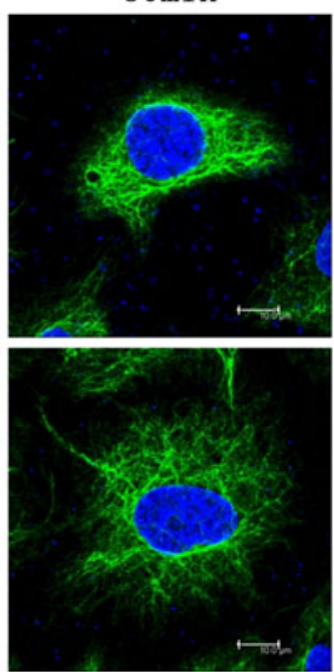

$1 \mathrm{~h}$
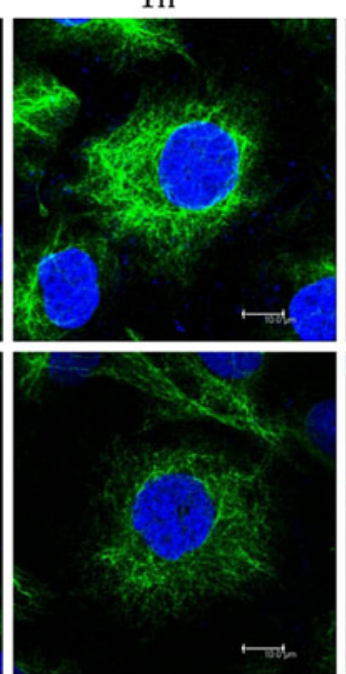

$8 \mathrm{~h}$
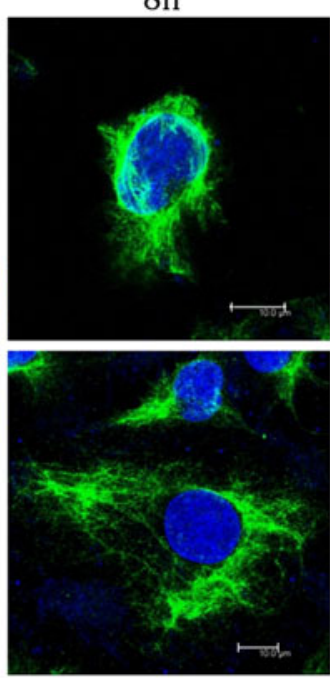

$24 \mathrm{~h}$
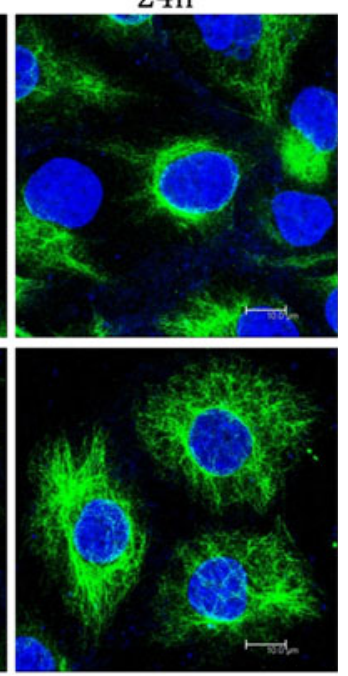

B

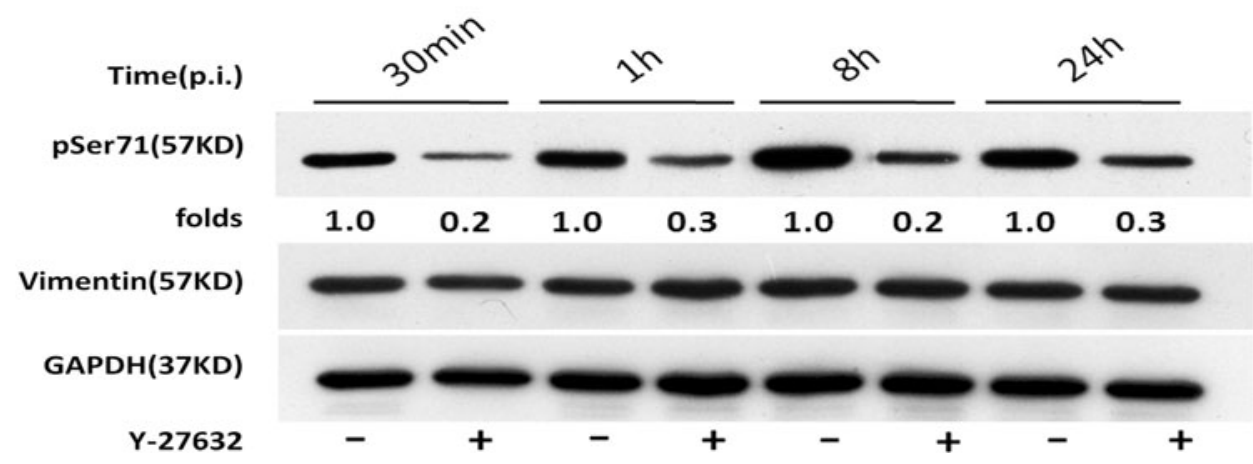

Fig. 4 Inactivation of ROCK with Y-27632 inhibited DENV2induced vimentin rearrangement and phosphorylation of vimentin at Ser71. ECV304 cells were pretreated with $10 \mu \mathrm{M}$ Y-27632 or mock treated for $1 \mathrm{~h}$ at $37^{\circ} \mathrm{C}$ prior to infection with DENV2. The cells were collected at different time points p.i. and then stained with the anti-vimentin antibody or analyzed by Western blotting with antibodies specific for Ser71-phosphorylated vimentin and vimentin, respectively. a Effect of ROCK on vimentin distribution.

DENV2 Infection Induced Phosphorylation of Vimentin, Followed by the Rearrangement of Vimentin IF Networks

The influence of DENV2 infection on the distribution of vimentin was also investigated with immunofluorescence co-localization staining. As shown in Fig. 2, vimentin IFs emanated from the perinuclear region and extended into the cytoplasm in mock-infected cells (Fig. 2a). However, in the infected cells, the collapsed vimentin network aggregated into dense structures at the perinuclear area and co-localized with NS1 (Fig. 2b, arrows). Furthermore, as it has previously been shown that the rearrangement of vimentin is driven by vimentin phosphorylation [11], we investigated the distribution of phosphorylated vimentin in DENV-infected cells. Interestingly, immunofluorescent staining with an anti-phospho-vimentin antibody showed a
Immunostaining images show that Y-27632 prevented the rearrangement of vimentin aggregates into dense structures at the perinuclear area in DENV2-infected cells at $30 \mathrm{~min}, 1 \mathrm{~h}, 8 \mathrm{~h}$, and $24 \mathrm{~h}$ p.i. Scale bars $10 \mu \mathrm{m}$. b Effect of ROCK on the phosphorylation of vimentin at Ser71. The results show that in Y-27632-treated cells, phosphorylation of vimentin at Ser71 was decreased at $30 \mathrm{~min}, 1 \mathrm{~h}, 8 \mathrm{~h}$, and $24 \mathrm{~h}$ p.i. Each blot represents data from a minimum of six separate experiments

similar alteration of the vimentin network compared to vimentin staining, implying that DENV2 may induce the rearrangement of vimentin by affecting its phosphorylation state (Fig. 2c).

\section{ROCK was Significantly Activated During DENV2} Infection

ROCK is a key serine/threonine kinase responsible for the phosphorylation and subsequent rearrangement of vimentin [12]. Therefore, ECV304 cells were infected with DENV2 at an MOI of 1, and lysates from different times p.i. were tested for ROCK activity. Compared to the mock-infected cells, we observed a 2.4-fold increase in ROCK activity in the DENV-infected cells at $30 \mathrm{~min}$ p.i., which then decreased to approximately 1.8 -fold greater than the mock at $1 \mathrm{~h}$ p.i. ROCK activity significantly increased again at 

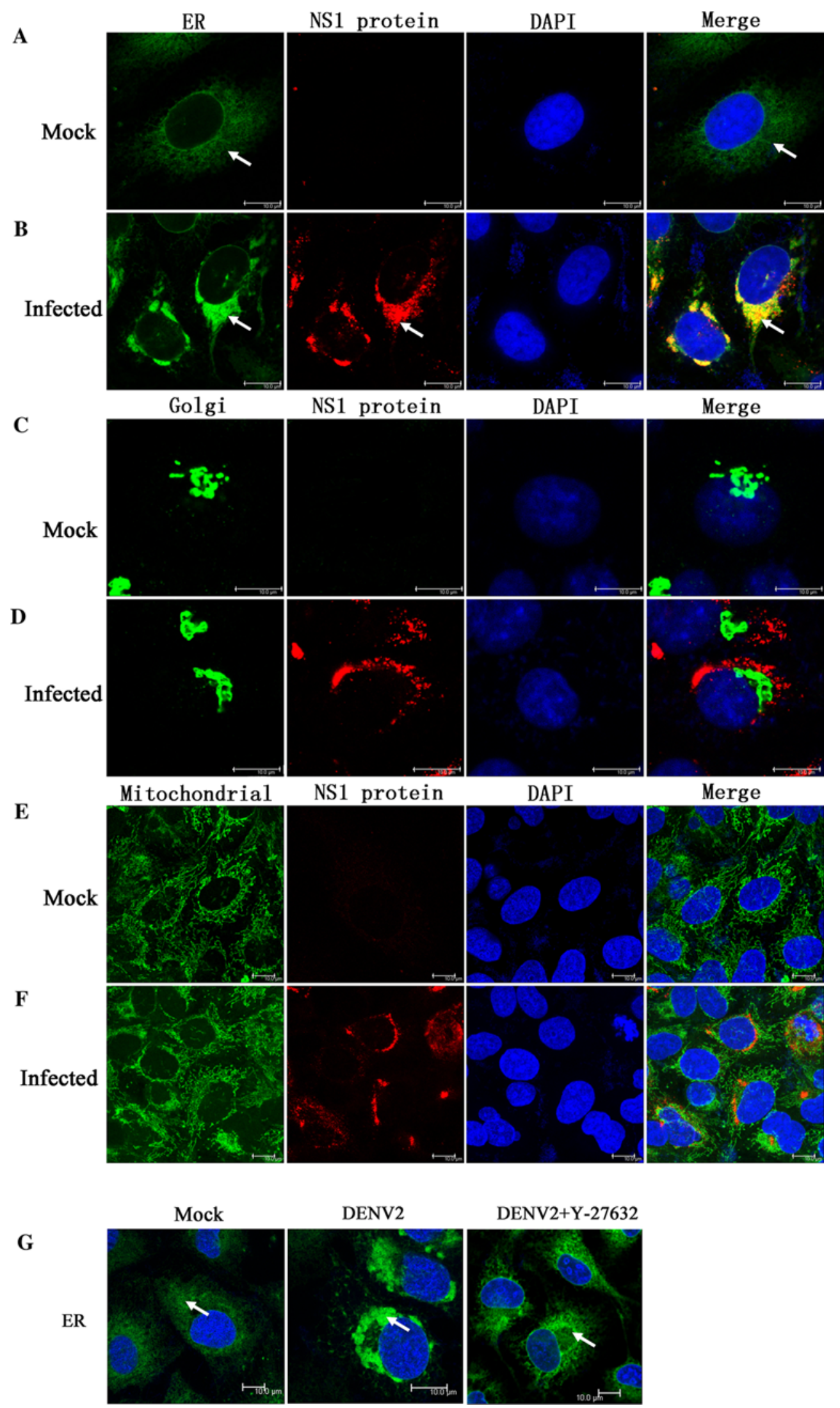
4ig. 5 Inactivation of ROCK with Y-27632 partially inhibited DENV2-induced ER rearrangement. Cells were infected for $24 \mathrm{~h}$ with DENV2. Viral replication sites were located using antibodies specific for the NS1 protein (red), and the ER was visualized using antibodies specific for calnexin (green). The Golgi complex was labeled with an anti-giantin antibody (green), and mitochondria were labeled with MitoTracker Green. Normal distribution of the ER (a), Golgi complex (c), and mitochondria (e) in uninfected cells is shown. In DENV2-infected cells, the ER staining redistributed to the perinuclear region and co-localized with DENV2 NS1 (b, arrows). No rearrangement of the Golgi apparatus or mitochondria was obvious in DENV2-infected cells (d, f). Y-27632 treatment partially blocked ER aggregation into dense structures (g). Scale bars $10 \mu \mathrm{m}$ (Color figure online)

$8 \mathrm{~h} \mathrm{p}$.i. and decreased thereafter, with approximately a 2.2fold increase at $8 \mathrm{~h}$ p.i., displaying an dynamic change with the progress of DENV2 infection. These data indicated that ROCK was significantly activated in the early stage of the vimentin rearrangement process (Fig. 3a), suggesting a putative important role for ROCK in modulating the rearrangement of vimentin induced by DENV infection. We next ask whether ROCK promotes the rearrangement of vimentin via upregulating vimentin phosphorylation during the infection process.

\section{Vimentin Phosphorylation at Ser71 Dynamically Changed During DENV2 Infection}

It has been reported that Ser71 is the most favored and unique phosphorylation site on vimentin for ROCK [13]. Therefore, it was necessary to determine the levels of Ser71-phosphorylated vimentin during DENV2 infection, which indicate the effect of ROCK on vimentin rearrangement. Accordingly, a quantitative protein analysis of vimentin and Ser71-phosphorylated vimentin was performed with Western blotting. As shown in Fig. 4b, despite no obvious change in total vimentin protein expression levels, Ser71-phosphorylated vimentin levels significantly increased in a time-dependent manner in DENV-infected cells. Compared to the mock-infected cells, DENV2 induced an increase (2.3-fold) in Ser71 phosphorylation as early as 15 min p.i., which peaked at 1 h p.i. (6.2-fold) and decreased thereafter, with increases of approximately 4.5and 4.1 -fold at 2 and $4 \mathrm{~h}$ p.i. Interestingly, vimentin Ser71 phosphorylation levels significantly increased again at $8 \mathrm{~h}$ p.i. and then decreased at $12 \mathrm{~h}$ p.i., with approximately 7.8-, 3.6-, and 4.6-fold increases relative to the mock at 8,12 , and $24 \mathrm{~h}$ p.i., respectively (Fig. $3 \mathrm{~b}$ ). These data suggested that activated ROCK may promote vimentin phosphorylation at Ser71, which may induce the rearrangement of vimentin caused by DENV infection. In addition, combined with the similar dynamic changes in ROCK activity during the progress of DENV2 infection, the dynamic changes in vimentin phosphorylation at Ser71 may reflect the fact that the level of phosphorylated vimentin is determined by the activation level of ROCK. Our next question was whether blocking ROCK activity could prevent vimentin phosphorylation at Ser71 and subsequently inhibit vimentin rearrangement induced by DENV2 infection.

Inactivation of ROCK with Y-27632 Inhibited DENV2Induced Vimentin Rearrangement and Phosphorylation of Vimentin at Ser71

The ROCK inhibitor Y-27632 was used to further characterize the role of ROCK in DENV2-induced vimentin rearrangement. Immunofluorescent staining revealed that compared to the mock group, Y-27632 pretreatment markedly blocks DENV2-induced rearrangement of vimentin in ECV304 cells, suggesting that ROCK plays an important role in DENV2-induced vimentin rearrangement (Fig. 4a).

Next, Y-27632 was used to determine the role of ROCK in the phosphorylation of vimentin at Ser71. As shown in Fig. 4b, Y-27632 pretreatment induced a significant and consistent decrease in the levels of Ser71 phosphorylation from $30 \mathrm{~min}$ to $24 \mathrm{~h}$ p.i. compared to the mock group. There was no significant difference in vimentin protein expression between DENV-infected groups with or without Y-27632 treatment (Fig. 4b). Taken together, these results demonstrated that DENV2-induced phosphorylation and rearrangement of vimentin may be dependent upon ROCK activation.

Inactivation of ROCK with Y-27632 Partially Inhibited DENV2-Induced ER Rearrangement During Virus Replication

It is generally believed that aside from vimentin rearrangement, large-scale changes in cellular organelles are also involved in the formation of viral factories in host cells, which may further benefit the virus's replication [14]. Accordingly, our next approach was to exam whether DENV infection induced the rearrangement of cellular membrane structures, such as the ER, Golgi apparatus, and mitochondria. Therefore, different organelle-specific markers were used to investigate the distribution of cellular membrane structures before and after DENV2 infection. DENV2 NS1 protein was used as a DENV RNA replication marker to visualize the co-localization of DENV and the ER, Golgi apparatus, and mitochondria at $24 \mathrm{~h}$ p.i. as previously described [15]. Immunofluorescent analysis showed that the normal ER network changed dramatically in DENV2-infected cells, that is, the ER moved to the perinuclear region, aggregated into dense structures, and co-localized with DENV2 NS1 (Fig. 5a, b, arrows). 
However, no similar changes were observed for the Golgi apparatus and mitochondria in DENV2-infected cells (Fig. 5c-f). This suggested that DENV2 infection causes the rearrangement of the preexisting ER network.

Interestingly, in a following experiment, pretreatment with the ROCK inhibitor Y-27632 partially blocked the rearrangement of the ER in DENV2-infected ECV304 cells (Fig. 5g, arrows). Immunofluorescent staining revealed that compared to the mock group, Y-27632 pretreatment partially blocked ER movement to the perinuclear region and aggregation into dense structures, suggesting that structural changes in the ER in DENV-infected cells may depend upon ROCK activation in a similar fashion to vimentin.

\section{Discussion}

In this study, we investigated how DENV2 infection leads to the reorganization of vimentin and the ER into dense structures at the perinuclear area. We found that DENV2 infection induced a marked change in the organization of the vimentin network, accompanying the entire process of the viral replication cycle. Ser71-phosphorylated vimentin displayed a similar distribution to total vimentin in DENV2-infected cells, forming a dense structure that also co-localized with DENV2 NS1 at the perinuclear area. Furthermore, DENV2 infection significantly induced ROCK activation and phosphorylation of vimentin at Ser71. Pretreatment with the ROCK inhibitor Y-27632 markedly prevented the rearrangement of vimentin and the increase in vimentin phosphorylation at Ser71 induced by DENV2 infection. Moreover, DENV2 led to ER redistribution in the perinuclear region of the host cells, which was partially blocked by pretreatment with Y-27632, suggesting that ROCK activation may be involved in the rearrangement of the ER during the DENV infection process.

It has been reported that vimentin rearrangement is important for the viral life cycle, including entry, transport, replication, and egress [16]. A perinuclear condensation of the vimentin IF network observed early in adenovirus infection appears to be a cytological marker for cytoplasmic transit of infectious virions within the infected cells [17]. Risco et al. [18] show that vimentin concentrates in the viral factories of vaccinia virus to participate in vial assembly. In addition, vimentin is also necessary for the trafficking of blue tongue virus to the cell surface [19], and foot and mouth disease virus modulates cellular vimentin for virus survival [20]. However, limited information is known about the role of vimentin in DENV infection thus far.

Our previous study revealed that DENV2 infection induces vimentin retraction from the cell periphery to the region surrounding the nucleus, suggesting that vimentin rearrangement is involved in DENV2 infection and replication. Recently, Kanlaya et al. further confirmed that vimentin interacts with heterogeneous nuclear ribonucleoproteins and dengue NS1 protein [10]. Although growing evidence indicates that vimentin is important for viral replication and release, the molecular mechanism linking DENV infection and vimentin rearrangement is still not well defined.

Accumulating evidence indicates that vimentin phosphorylation plays an important role in the rearrangement of vimentin [21]. Vimentin architecture is maintained in a dynamic state that is regulated by the balance between phosphorylation and dephosphorylation [10]. It has been shown that vimentin phosphorylation in association with vimentin disassembly and spatial reorganization occurs during mitosis or in response to extracellular stimulation [11]. Phosphorylation of vimentin may also underlie the mechanism for regulating cage formation around virus assembly sites during ASFV and frog virus 3 infection, which may provide a scaffold to prevent diffusion of viral components into the cytoplasm [5, 6].

Here, we showed that DENV2 infection induced phosphorylation of vimentin, followed by the rearrangement of vimentin. Furthermore, the severe perinuclear condensation of phosphorylated vimentin and the co-localization of vimentin with DENV NS1 both indicate a potential relationship between vimentin phosphorylation and the formation of DENV replication sites. It is possible that DENV2 infection induces a qualitative change in vimentin, and this change may coincide with the reorganization of vimentin around the viral assembly sites, but further studies are needed to elucidate the relationship between vimentin rearrangement and the formation of DENV replication sites. It should be noted that the fluctuation of the level of Ser71-phosphorylated vimentin with the stages of DENV-2 infection may reflect the fact that dengue virus exploits phosphorylated vimentin in varying degrees for various events in the viral life cycle.

ROCK is a key serine/threonine kinase responsible for the phosphorylation and subsequent rearrangement of vimentin [21]. In vitro, Ser71 was the most favored and unique phosphorylation site for ROCK on vimentin [13]. Here, we found that DENV2 infection induces a significant activation of ROCK, as well as an increase in vimentin phosphorylation at Ser71. Furthermore, inactivation of ROCK with Y-27632 significantly inhibited DENV2induced vimentin phosphorylation at Ser71 and the rearrangement of vimentin. These results suggest that ROCK may play an important role in DENV-induced vimentin rearrangement by modulating the phosphorylation state of vimentin. In addition, our recent data show that RhoA, the key upstream molecule of ROCK, is also activated during DENV2 infection (unpublished, data not shown). Further 
work is ongoing in our laboratory to test our hypothesis that the RhoA/ROCK pathway is involved in the progress of DENV infection and replication. However, it should be noted that vimentin is an excellent substrate for a number of kinases in vitro, and multiple vimentin phosphorylation sites have been identified. For example, protein kinase A primarily phosphorylates vimentin on sites Ser38 and Ser72, and CaM kinase II phosphorylates vimentin at Ser38 and Ser82 [21, 22]. Thus, it is necessary to explore the potential role of other important kinases in the mechanism of DENV-induced vimentin rearrangement.

It has been shown that the rearrangement of the ER provides the virus with an initial membranous platform to establish a productive infection [23]. Additionally, the ER localizes and concentrates preexisting cellular proteins required for viral translation and replication [24]. The interaction between the ER chaperone calnexin and the viral glycoproteins prM, E, and NS1 has also been revealed by co-immunoprecipitation [25]. Recently, a study of DENV2 using electron microscopy and tomography found that DENV modifies the ER membrane structure to promote replication and efficient encapsulation of the genome into progeny viruses [15]. This was further confirmed in the present study: DENV2 infection induced a significant rearrangement of the ER around the perinuclear region, colocalizing with DENV2 NS1. Of note, a structural interaction between the ER and vimentin has also been documented [26]. Considering that vimentin plays a significant role in supporting and anchoring the position of the organelles, such as the nucleus, ER, and mitochondria, in the cytosol, it is possible that the rearrangement of vimentin may also be involved in the redistribution of the ER around the perinuclear region during DENV infection, which may promote the formation of DENV replication sites. Interestingly, we showed that Y-27632 pretreatment partially blocks the collapse of the ER into a region around the nucleus, indicating that ROCK activation may also be involved in DENV-induced ER rearrangement. The present results support the possibility that ROCK activation induces both vimentin rearrangement and ER redistribution around the perinuclear region, which may play a structural role in anchoring the viral replication sites.

In conclusion, we demonstrated that DENV2 infection induces the rearrangement of vimentin and the ER around the perinuclear region by activating ROCK. The ROCK phosphorylation of vimentin at Ser71 may play an important role in vimentin rearrangement during DENV infection. In the future, it will be important to investigate the influence of the rearrangement of vimentin and the ER on the formation of DENV replication sites and the important role of the RhoA/ROCK pathway in this process. Additionally, it is of interest to elucidate the relationship between vimentin and ER rearrangement during DENV2 infection.
Acknowledgments This work was supported by grants 30972596 and 31070811 from the National Natural Science Foundation of China, 2012ZX09103301-038 from the New Drug Development Project of China, and 2012XJQ05 and 2009XQN10 from the Third Military Medical University.

\section{Conflict of interest None.}

Open Access This article is distributed under the terms of the Creative Commons Attribution License which permits any use, distribution, and reproduction in any medium, provided the original author(s) and the source are credited.

\section{References}

1. Chen, S. T., Lin, Y. L., Huang, M. T., Wu, M. F., Cheng, S. C., Lei, H. Y., et al. (2008). CLEC5A is critical for dengue-virusinduced lethal disease. Nature, 453, 672-676.

2. Döhner, K., \& Sodeik, B. (2005). The role of the cytoskeleton during viral infection. Current Topics in Microbiology and Immunology, 285, 67-108.

3. Radtke, K., Döhner, K., \& Sodeik, B. (2006). Viral interactions with the cytoskeleton: A hitchhiker's guide to the cell. Cellular Microbiology, 8, 387-400.

4. Kim, J. K., Fahad, A. M., Shanmukhappa, K., \& Kapil, S. (2006). Defining the cellular target(s) of porcine reproductive and respiratory syndrome virus blocking monoclonal antibody 7G10. Journal of Virology, 80, 689-696.

5. Murti, K. G., Goorha, R., \& Klymkowsky, M. W. (1988). A functional role for intermediate filaments in the formation of frog virus 3 assembly sites. Virology, 162, 264-269.

6. Stefanovic, S., Windsor, M., Nagata, K. I., Inagaki, M., \& Wileman, T. (2005). Vimentin rearrangement during African swine fever virus infection involves retrograde transport along microtubules and phosphorylation of vimentin by calcium calmodulin kinase II. Journal of Virology, 79, 11766-11775.

7. Karczewski, M. K., \& Strebel, K. (1996). Cytoskeleton association and virion incorporation of the human immunodeficiency virus type 1 Vif protein. Journal of Virology, 70, 494-507.

8. Chen, W., Gao, N., Wang, J. L., Tian, Y. P., Chen, Z. T., \& An, J. (2008). Vimentin is required for dengue virus serotype 2 infection but microtubules are not necessary for this process. Archives of Virology, 153, 1777-1781.

9. Kanlaya, R., Pattanakitsakul, S. N., Sinchaikul, S., Chen, S. T., \& Thongboonkerd, V. (2010). Vimentin interacts with heterogeneous nuclear ribonucleoproteins and dengue nonstructural protein 1 and is important for viral replication and release. Molecular BioSystems, 6, 795-806.

10. Nakamura, Y., Hashimoto, R., Amano, M., Nagata, K., Matsumoto, N., Goto, H., et al. (2000). Localized phosphorylation of vimentin by Rho-kinase in neuroblastoma N2a cells. Genes to Cells, 5, 823-837.

11. Li, Q. F., Spinelli, A. M., Wang, R., Anfinogenova, Y., Singer, H. A., \& Tang, D. D. (2006). Critical role of vimentin phosphorylation at Ser-56 by 21 -activated kinase in vimentin cytoskeleton signaling. Journal of Biological Chemistry, 281, 34716-34724.

12. Sin, W. C., Chen, X. Q., Leung, T., \& Lim, L. (1998). RhoAbinding kinase a translocation is facilitated by the collapse of the vimentin intermediate filament network. Molecular and Cellular Biology, 18, 6325-6339.

13. Goto, H., Kosako, H., Tanabe, K., Yanagida, M., Sakurai, M., Amano, M., et al. (1998). Phosphorylation of vimentin by Rhoassociated kinase at a unique amino-terminal site that is 
specifically phosphorylated during cytokinesis. Journal of Biological Chemistry, 273, 11728-11736.

14. Novoa, R. R., Calderita, G., Arranz, R., Fontana, J., Granzow, H., \& Risco, C. (2005). Virus factories: Associations of cell organelles for viral replication and morphogenesis. Biology of the Cell, 97, 147-172.

15. Welsch, S., Miller, S., Romero-Brey, I., Merz, A., Bleck, C. K., Walther, P., et al. (2009). Composition and three-dimensional architecture of the dengue virus replication and assembly sites. Cell Host and Microbe, 5, 365-375.

16. Sripada, S., \& Dayarai, C. (2010). Viral interactions with intermediate filaments: Paths less explored. Cell Health and Cytoskeleton, 2, 1-7.

17. Defer, C., Belin, M. T., Caillet-Boudin, M. L., \& Boulanger, P. (1990). Human adenovirus-host cell interactions: Comparative study with members of subgroups B and C. Journal of Virology, 64, 3661-3673.

18. Risco, C., Rodríguez, J. R., López-Iglesias, C., Carrascosa, J. L., Esteban, M., \& Rodríguez, D. (2002). Endoplasmic reticulumGolgi intermediate compartment membranes and vimentin filaments participate in vaccinia virus assembly. Journal of Virology, $76,1839-1855$.

19. Bhattacharya, B., Noad, R. J., \& Roy, P. (2007). Interaction between Bluetongue virus outer capsid protein VP2 and vimentin is necessary for virus egress. Virology of Journal, 4, 7.
20. Gladue, D. P., O’Donnell, V., Baker-Branstetter, R., Holinka, L. G., Pacheco, J. M., Sainz, I. F. et al. (2013) Foot-and-mouth disease virus modulates cellular vimentin for virus survival. Journal of virology.

21. Yasui, Y., Goto, H., Matsui, S., Manser, E., Lim, L., Nagata, Ki, et al. (2001). Protein kinases required for segregation of vimentin filaments in mitotic process. Oncogene, 20, 2868-2876.

22. Satelli, A., \& Li, S. (2011). Vimentin as a potential molecular target in cancer therapy. Cellular and Molecular Life Sciences, 68, 3033-3046.

23. Novoa, R. R., Calderita, G., Arranz, R., Fontana, J., Granzow, H., \& Risco, C. (2005). Virus factories: Associations of cell organelles for viral replication and morphogenesis. Biology of the Cell, 97, 147-172.

24. Netherton, C., Moffat, K., Brooks, E., \& Wileman, T. (2007). A guide to viral inclusions, membrane rearrangements, factories, and viroplasm produced during virus replication. Advances in Virus Research, 70, 101-182.

25. Wu, S. F., Lee, C. J., Liao, C. L., Dwek, R. A., Zitzmann, N., \& Lin, Y. L. (2002). Antiviral effects of an iminosugar derivative on flavivirus infections. Journal of Virology, 76, 3596-3604.

26. Katsumoto, T., Mitsushima, A., \& Kurimura, T. (1990). The role of the vimentin intermediate filaments in rat $3 \mathrm{Y} 1$ cells elucidated by immunoelectron microscopy and computer-graphic reconstruction. Biology of the Cell, 68, 139-146. 